\title{
Dehalogenimonas spp. can Reductively Dehalogenate High Concentrations of 1,2-Dichloroethane, 1,2-Dichloropropane, and 1,1,2-Trichloroethane
}

\author{
Andrew D Maness ${ }^{1}$, Kimberly S Bowman ${ }^{1,2}$, Jun Yan ${ }^{1,4}$, Fred A Rainey ${ }^{2,3}$ and William M Moe ${ }^{1 *}$
}

\begin{abstract}
The contaminant concentrations over which type strains of the species Dehalogenimonas alkenigignens and Dehalogenimonas lykanthroporepellens were able to reductively dechlorinate 1,2-dichloroethane (1,2-DCA), 1,2-dichloropropane (1,2-DCP), and 1,1,2-trichloroethane (1,1,2-TCA) were evaluated. Although initially isolated from an environment with much lower halogenated solvent concentrations, D. alkenigignens IP3-3 ${ }^{\top}$ was found to reductively dehalogenate chlorinated alkanes at concentrations comparable to D. lykanthroporepellens BL-DC-9 . Both species dechlorinated 1,2-DCA, 1,2-DCP, and 1,1,2-TCA present at initial concentrations at least as high as 8.7, 4.0 , and $3.5 \mathrm{mM}$, respectively. The ability of Dehalogenimonas spp. to carry out anaerobic reductive dechlorination even in the presence of high concentrations of chlorinated aliphatic alkanes has important implications for remediation of contaminated soil and groundwater.
\end{abstract}

Keywords: Bioremediation, Chlorinated alkanes, Dehalogenimonas, Reductive dechlorination, Dehalogenation

\section{Introduction}

In industry, polychlorinated ethanes and propanes are used as solvents, degreasing agents, and paint removers; they are also globally produced on a massive scale as intermediates during production of other industrially important chemicals (De Wildeman and Verstraete, 2003; Field and Sierra-Alvarez, 2004). Due to spills and past disposal methods, these chlorinated organic compounds are prevalent groundwater and soil contaminants. For example, 1,2-dichloroethane (1,2-DCA) is present in at least 570 current or former Superfund sites (ATSDR 2001), and 1,2-dichloropropane (1,2-DCP) is present at more than 100 Superfund sites (Fletcher et al., 2009). The prevalence of these polychlorinated alkanes as environmental contaminants is of concern because of their known or suspected toxicity and/or carcinogenicity (ATSDR, 2001; 1989).

\footnotetext{
* Correspondence: moemwil@lsu.edu

'Department of Civil and Environmental Engineering, Louisiana State University, 3513B Patrick Taylor Hall, Baton Rouge, LA 70803, USA Full list of author information is available at the end of the article
}

Anaerobic reductive dechlorination, a process in which microorganisms utilize chlorinated organics as electron acceptors, represents a potentially viable method for cleanup of many contaminated sites (Christ et al., 2005; Fennell et al., 2001; He et al., 2005; Major et al., 2002). Previous studies on reductive dechlorination of halogenated alkanes have generally been conducted in a relatively narrow range of low (e.g., 0.1 to $0.5 \mathrm{mM}$ ) contaminant concentrations (Chen et al., 1996; De Wildeman et al., 2003; Fletcher et al., 2009; Grostern and Edwards, 2006, 2009; Lorah and Olsen, 1999; Maymó-Gatell et al., 1999; Yan et al., 2009a). Contaminant concentrations considerably higher than this range are present at some sites, however, particularly in areas where pollutants remain in the subsurface as dense nonaqueous-phase liquids (DNAPLs) (Bowman et al., 2006; Marzorati et al., 2007; Yan et al., 2009b).

Among the limited number of microbes known to anaerobically reductively dehalogenate polychlorinated ethanes and propanes are strains of Dehalogenimonas lykanthroporepellens (Moe et al., 2009; Yan et al., 2009a) and Dehalogenimonas alkenigignens (Bowman et al., 
2012). These species cluster in the phylum Chloroflexi, related to but distinct from organohalide respiring Dehalococcoides strains (Bowman et al., 2012; Löffler et al., 2012; Moe et al., 2009). Strains of both Dehalogenimonas species reductively dehalogenate 1,2-DCA, 1,2-DCP, and 1,1,2-TCA via dichloroelimination reactions with $\mathrm{H}_{2}$ as an electron donor, forming final products of ethene, propene, and vinyl chloride, respectively (Bowman et al., 2012; Yan et al., 2009a).

Previously reported studies of Dehalogenimonas strains were conducted only at initial chlorinated solvent concentrations of $0.5 \mathrm{mM}$ (Bowman et al., 2012; Yan et al., 2009a). Research reported here was aimed at evaluating the solvent concentration ranges over which D. lykanthroporepellens and D. alkenigignens can reductively dechlorinate 1,2-DCA, 1,2-DCP, and 1,1,2-TCA in order to assess their suitability for bioremediation of high contaminant concentrations.

\section{Materials and methods}

Experiments were carried out in $25 \mathrm{~mL}$ glass serum bottles (Wheaton) sealed with butyl rubber stoppers and aluminum crimp caps. Each serum bottle contained 10 $\mathrm{mL}$ titanium-citrate reduced anaerobic basal medium prepared as described by (Moe et al. 2009) except that 5 $\mathrm{mM}$ acetate was replaced with $0.05 \mathrm{mM}$ each of acetate, pyruvate, and lactate. The $15 \mathrm{~mL}$ gas headspace was comprised of $\mathrm{H}_{2} / \mathrm{N}_{2}(80 \% / 20 \%$, v/v). Replicate serum bottles were spiked with neat, filter sterilized 1,2-DCA (>99.8\% purity, Sigma Aldrich, St. Louis, MO), 1,2-DCP (99\%, Sigma Aldrich, St. Louis, MO), or 1,1,2-TCA (96\%, Sigma Aldrich, St. Louis, MO) to achieve target initial aqueous phase concentrations ranging from 0.5 to 15 $\mathrm{mM}$ after dissolution and equilibration.

Each serum bottle received $0.3 \mathrm{~mL}$ inoculum $(3 \% \mathrm{v} / \mathrm{v})$ of $D$. alkenigignens strain IP3-3 ${ }^{\mathrm{T}}\left(=\mathrm{JCM} 17062^{\mathrm{T}}=\mathrm{NRRL}\right.$ $\left.\mathrm{B}-59545^{\mathrm{T}}\right)$ or D. lykanthroporepellens strain BL-DC-9 ${ }^{\mathrm{T}}$ $\left(=\mathrm{JCM} 15061^{\mathrm{T}}=\right.$ ATCC BAA- $\left.1523^{\mathrm{T}}\right)$ previously grown on $1,2-\mathrm{DCP}$. Incubation was in the dark at $30^{\circ} \mathrm{C}$ without shaking. Triplicate bottles were sacrificed at time zero and after eight weeks incubation for analysis of chlorinated solvents and potential degradation products. To account for potential abiotic reactions, triplicate negative controls prepared in the same manner as inoculated bottles but without bacterial addition were incubated under identical conditions.

Chlorinated solvents and degradation products were measured using an HP model 6890 gas chromatograph (GC) equipped with a flame ionization detector and GS-GasPro capillary column $(60 \mathrm{~m} \times 0.32 \mathrm{~mm}$ I.D., J\&W P/N 113-4362) as described previously (Yan et al., 2009a). Gas headspace samples collected in $100 \mu \mathrm{L}$ gastight glass syringes (Hamilton, Baton Rouge, LA) were introduced to the $\mathrm{GC}$ via direct injection. Aqueous samples $(500 \mu \mathrm{L})$ were introduced to the GC via a Tekmar 2016/3000 purge and trap autosampler and concentrator. Both gas-headspace and aqueous-phase aliquots were analyzed for each sample bottle.

Hydrogen concentrations in the gas headspace were measured using an SRI Instruments model 310 gas chromatograph (Torrence, CA) equipped with a thermal conductivity detector and molecular sieve column (Alltech Molesieve 5A 80/100) as described previously (van Ginkel et al., 2001).

\section{Results}

The quantity of the dechlorination product determined at the end of the eight week incubation period as a function of initial aqueous-phase 1,2-DCA, 1,-DCP, and 1,1,2-TCA is shown in Figures 1, 2 and 3 respectively.

The production of ethene (Figure 1) coupled with 1,2-DCA disappearance in the inoculated bottles is consistent with the 1,2-DCA dihaloelimination degradation pathway reported previously for $D$. alkenigignens IP3- ${ }^{\mathrm{T}}$ and D. lykantroporepellens BL-DC- $9^{\mathrm{T}}$ in tests conducted with initial 1,2-DCA concentrations of $0.5 \mathrm{mM}$ in serum bottles containing $\mathrm{H}_{2}$ at an initial concentration of $10 \%$ $\mathrm{v} / \mathrm{v}$ (as opposed to the $80 \% \mathrm{v} / \mathrm{v}$ employed in the present study) (Bowman et al., 2012; Yan et al., 2009a). Trace levels of 1 -chloroethane $(<0.3 \mu \mathrm{mol} / \mathrm{bottle})$ were detected at comparable levels in both inoculated bottles and in uninoculated abiotic controls (data not shown) and small amounts of ethene $(<0.7 \mu \mathrm{mol} / \mathrm{bottle})$ were detected in abiotic negative controls (Figure 1) indicating that some abiotic 1,2-DCA transformation occurred in the anaerobic medium employed here, but the amount was negligible. The sum of parent compound (i.e., 1,2-DCA) plus daughter product (i.e., ethene and 1-chloroethane) in replicate serum bottles inoculated with the bacterial strains ranged from $74-107 \%$ of the mass determined in abiotic negative controls (average $89 \%)$. Dechlorination was essentially complete $(<1 \%$ of the starting 1,2-DCA remaining) at the end of the eight week incubation period for serum bottles supplemented with 1,2-DCA at initial concentrations less than $3.16 \pm 0.05 \mathrm{mM}$ and $1.48 \pm 0.03 \mathrm{mM}$ (mean \pm standard deviation) for D. alkenigignens IP3- $3^{\mathrm{T}}$ and D. lykanthroporepellens $\mathrm{BL}-\mathrm{DC}-9^{\mathrm{T}}$, respectively (Figure 1 ). At higher initial 1,2-DCA concentrations (at and to the right of concentrations denoted by arrows in Figure 1), untransformed 1,2-DCA remained at the end of the eight week incubation in amounts increasing with increasing initial 1,2-DCA concentration.

The quantity of ethene observed increased with increasing initial 1,2-DCA concentration in the range of 0.5 to approximately $4 \mathrm{mM}$ (maximum ethene observed in bottles containing initial 1,2-DCA concentrations of $4.03 \pm 0.09$ and $4.08 \pm 0.16 \mathrm{mM}$ for $D$. alkenigignens IP3- 

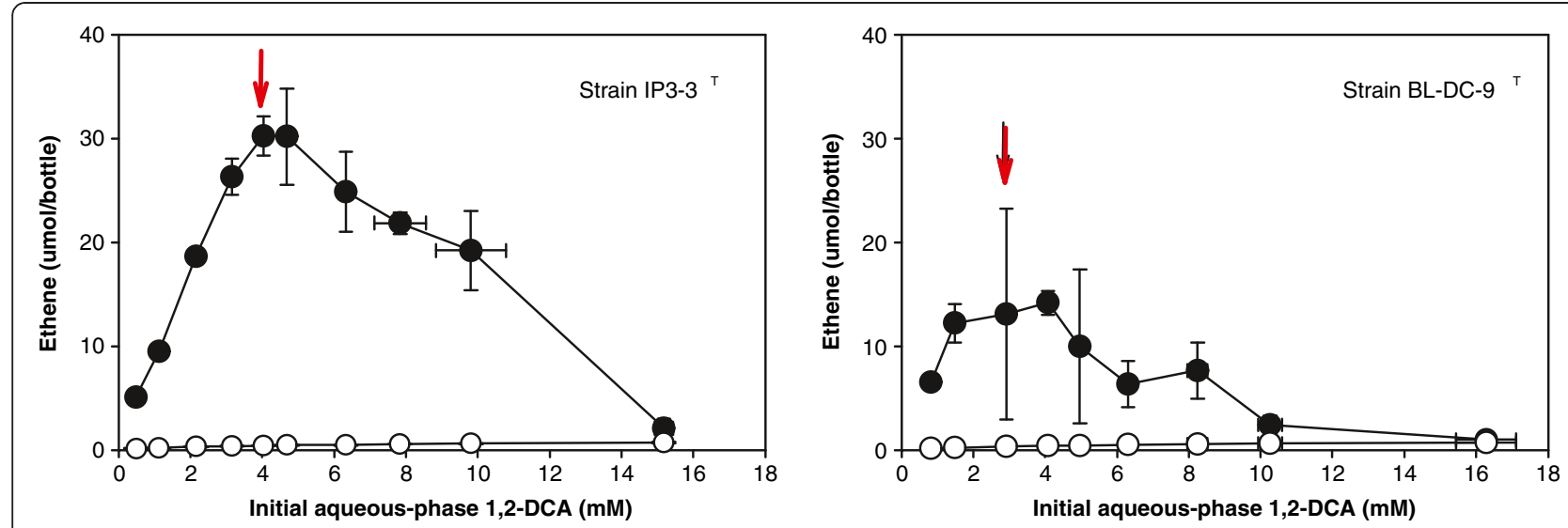

Figure 1 Experimentally measured ethene production as a function of initial aqueous-phase 1,2-DCA concentration after eight-weeks incubation of $D$. alkenigignens IP3-3 $3^{\top}$ (left) and $D$. lykanthroporepellens BL-DC-9 ${ }^{\top}$ (right). Filled symbols indicate average of replicate bottles inoculated with bacterial strains. Open symbols indicate average of replicate uninoculated negative control bottles. Bars represent one standard deviation. Arrows denote concentration at and above which $>1 \%$ of the starting 1,2-DCA remained at the end of the incubation period.

$3^{\mathrm{T}}$ and D. lykanthroporepellens BL-DC-9 ${ }^{\mathrm{T}}$, respectively) and then decreased at higher initial 1,2-DCA concentrations. The decrease in ethene production as 1,2-DCA concentrations increased indicates that sufficiently high 1,2-DCA concentrations can inhibit dechlorination activity of both Dehalogenimonas spp. Biologically mediated 1,2-DCA reductive dechlorination, however, was observed in serum bottles with initial 1,2-DCA concentrations as high as $9.81 \pm 0.98$ and $8.69 \pm 0.26 \mathrm{mM}$ for D. alkenigignens IP3- $3^{\mathrm{T}}$ and D. lykanthroporepellens BLDC- $9^{\mathrm{T}}$, respectively. At higher initial 1,2-DCA concentrations, small amounts of ethene were also detected, but in amounts that were not statistically different from abiotic negative controls at a confidence level of $95 \%$.

The production of propene (Figure 2) coupled with $1,2-\mathrm{DCP}$ dechlorination in the inoculated bottles is consistent with the previously reported tests conducted with $0.5 \mathrm{mM} 1,2-\mathrm{DCP}$ in serum bottles with $10 \% \mathrm{v} / \mathrm{v} \mathrm{H}_{2}$ in the gas headspace (Bowman et al., 2012; Yan et al., 2009a). Trace levels of 1 -chloropropane $(<0.03 \mu \mathrm{mol} /$ bottle) were detected in inoculated bottles and uninoculated abiotic controls (data not shown), and propene was detected in relatively minute quantities $(<0.13 \mu \mathrm{mol} /$ bottle) in abiotic negative controls (Figure 2), indicating small amounts of abiotic 1,2-DCP transformation. The sum of parent chlorinated solvent (i.e., 1,2-DCP) and daughter products (i.e., propene and 1-chloropropane) in replicate bottles inoculated with the bacterial strains ranged from $74-131 \%$ of the mass determined in abiotic negative controls (average 95\%). When provided with 1,2-DCP at initial aqueous-phase concentrations less than $3.19 \pm 0.20 \mathrm{mM}$ and $2.14 \pm 0.12 \mathrm{mM}$, dechlorination
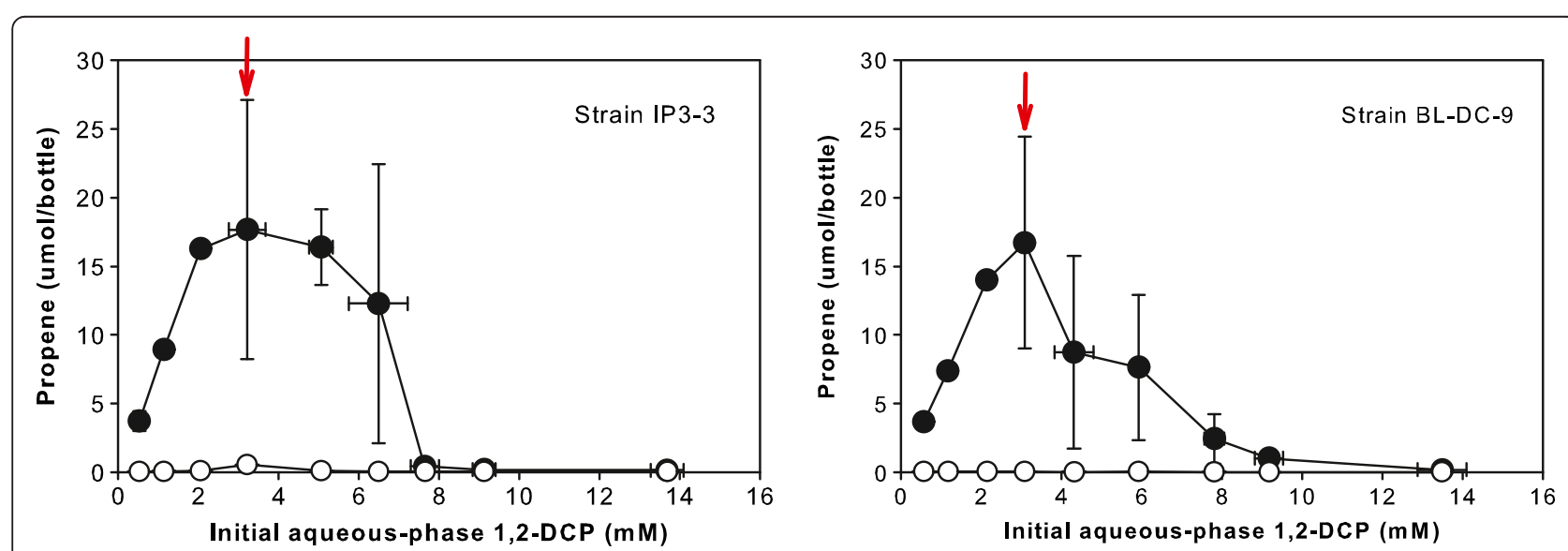

Figure 2 Experimentally measured propene production as a function of initial aqueous-phase 1,2-DCP concentration after eight-weeks incubation of $D$. alkenigignens IP3-3 $3^{\top}$ (left) and $D$. lykanthroporepellens BL-DC-9 $9^{\top}$ (right). Filled symbols indicate average of replicate bottles inoculated with bacterial strains. Open symbols indicate average of replicate uninoculated negative control bottles. Bars represent one standard deviation. Arrows denote concentration at and above which $>1 \%$ of the starting 1,2-DCP remained at the end of the incubation period. 

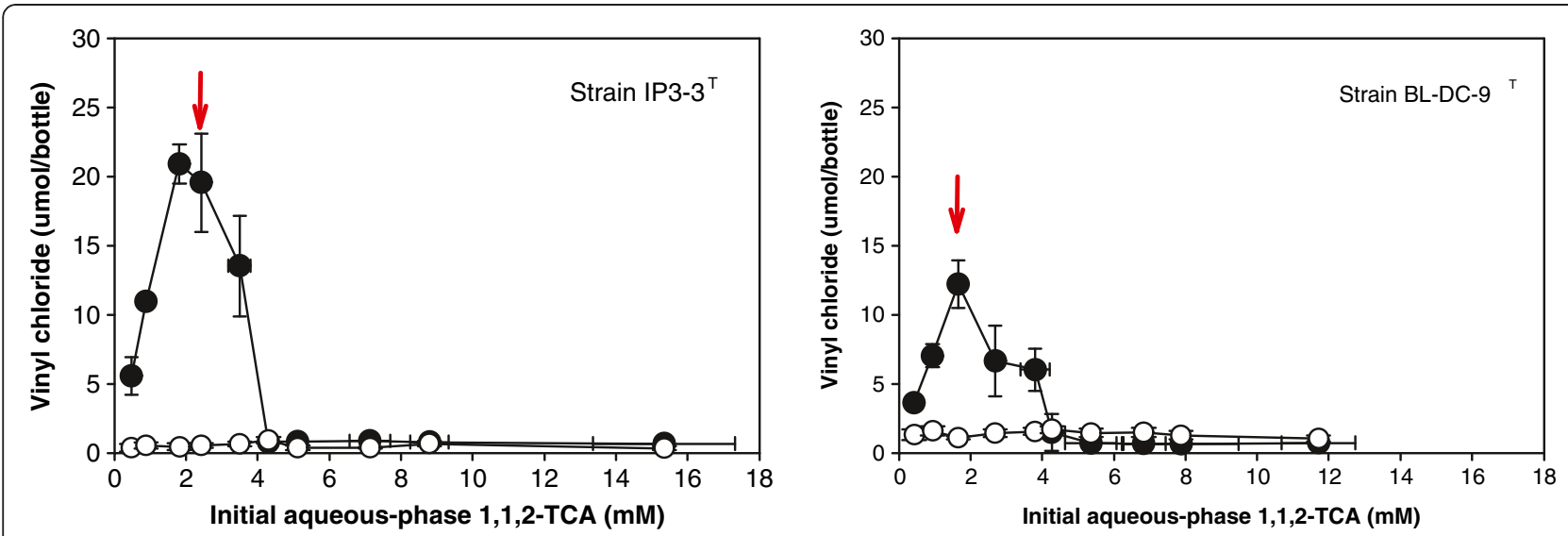

Figure 3 Experimentally measured vinyl chloride production as a function of initial aqueous-phase 1,1,2-TCA concentration after eight-weeks incubation of $D$. alkenigignens IP3-3 $3^{\top}$ (left) and $D$. lykanthroporepellens BL-DC-9 ${ }^{\top}$ (right). Filled symbols indicate average of replicate bottles inoculated with bacterial strains. Open symbols indicate average of replicate uninoculated negative control bottles. Bars represent one standard deviation. Arrows denote concentration at and above which $>1 \%$ of the starting 1,1,2-TCA remained at the end of the incubation period.

of 1,2-DCP to a final product of propene was essentially complete in bottles inoculated with $D$. alkenigignens IP3-3 ${ }^{\mathrm{T}}$ and D. lykanthroporepellens BL-DC-9 ${ }^{\mathrm{T}}$, respectively, with $<1 \%$ of the starting $1,2-\mathrm{DCP}$ remaining at the end of the eight week incubation period (Figure 2). At higher initial 1,2-DCP concentrations (denoted by arrows in Figure 2), 1,2-DCP remained at the end of eight weeks in amounts increasing with increasing initial 1,2-DCP concentration.

Similar to what was observed with 1,2-DCA, the quantity of propene formed from 1,2-DCP dechlorination increased at initial 1,2-DCP concentrations ranging from 0.5 to roughly $3 \mathrm{mM}$ (maximum propene was observed in bottles containing initial 1,2-DCP concentrations of $3.21 \pm 0.46$ and $3.08 \pm 0.05 \mathrm{mM}$ for $D$. alkenigignens IP3$3^{\mathrm{T}}$ and D. lykanthroporepellens BL-DC-9 ${ }^{\mathrm{T}}$, respectively) and then decreased at higher initial 1,2-DCP concentrations. This indicates that beyond a certain threshold, as was observed with 1,2-DCA, 1,2-DCP became inhibitory to dechlorination activity. Nevertheless, 1,2-DCP reductive dechlorination was observed in serum bottles with initial 1,2-DCP concentrations as high as $5.05 \pm 0.29$ and $4.02 \pm 0.09 \mathrm{mM}$ for $D$. alkenigignens IP3-3 ${ }^{\mathrm{T}}$ and $D$. lykanthroporepellens BL-DC-9 ${ }^{\mathrm{T}}$, respectively. At higher initial 1,2-DCP concentrations, propene was also detected, but in amounts that were not statistically different from abiotic negative controls at a $95 \%$ confidence level.

In contrast to the relatively high concentrations of 1,2DCP that were dechlorinated by Dehalogenimonas spp. in the present study, (Löffler et al. 1997) reported that 1,2-DCP dechlorination by an undefined mixed culture derived from Red Cedar Creek sediment (Michigan, USA) was completely inhibited when 1,2-DCP was supplied in amounts corresponding to an aqueous phase concentration of roughly $0.9 \mathrm{mM}$ or higher. D. alkenigignens $\mathrm{IP} 3-3^{\mathrm{T}}$ and D. lykanthroporepellens BL-DC-9 ${ }^{\mathrm{T}}$ may be better suited to degradation of higher 1,2-DCP concentrations than other microbial populations studied previously.

Vinyl chloride production (Figure 3) coupled with $1,1,2$-TCA dechlorination in the inoculated bottles is consistent with the previously reported tests conducted

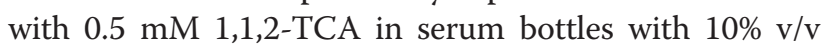
$\mathrm{H}_{2}$ in the gas headspace (Bowman et al., 2012; Yan et al., 2009a). Low levels of 1,2-DCA $(<1.5 \mu \mathrm{mol} /$ bottle $)$ were observed in both inoculated bottles and uninoculated negative controls (data not shown), and small quantities vinyl chloride $(<1 \mu \mathrm{mol} /$ bottle $)$ were observed in abiotic negative controls (Figure 3), indicating some abiotic 1,1,2-TCA transformation but in comparatively small amounts. The sum of the parent solvent (i.e., 1,1,2-TCA) and the daughter products (i.e., 1,2-DCA and vinyl chloride) in replicate bottles inoculated with the bacterial strains ranged from $74-146 \%$ of the mass determined in abiotic negative controls (average 99\%). Dechlorination was essentially complete ( $<1 \% 1,1,2$-TCA remaining) after 8 weeks incubation when $D$. alkenigignens IP3-3 ${ }^{\mathrm{T}}$ and D. lykanthroporepellens BL-DC- ${ }^{\mathrm{T}}$ were supplied with initial 1,1,2-TCA aqueous-phase concentrations below $2.42 \pm 0.22 \mathrm{mM}$ and $1.65 \pm 0.03 \mathrm{mM}$, respectively (Figure 3). At higher initial concentrations, untransformed 1,1,2-TCA remained at the end of the incubation period.

Similar to what was observed with 1,2-DCA and 1,2DCP, the quantity of vinyl chloride formed from 1,1,2TCA dechlorination increased at initial 1,1,2-TCA concentrations ranging from 0.5 to roughly $2 \mathrm{mM}$ and then decreased at higher initial 1,1,2-TCA concentrations 
(Figure 3). Maximum vinyl chloride concentrations were observed in bottles containing initial 1,1,2-TCA concentrations of $1.82 \pm 0.18$ and $1.65 \pm 0.03 \mathrm{mM}$ for D. alkenigignens IP3- ${ }^{\mathrm{T}}$ and D. lykanthroporepellens BL-DC- $9^{\mathrm{T}}$, respectively. This indicates that beyond a certain threshold, as was observed with 1,2-DCA and 1,2-DCP, 1,1,2-TCA became inhibitory to dechlorination activity. Nevertheless, biologically mediated 1,1,2-TCA reductive dechlorination was observed in serum bottles with initial 1,1,2-TCA concentrations as high as $3.49 \pm 0.31$ and $3.80 \pm 0.42 \mathrm{mM}$ for $D$. alkenigignens IP3$3^{\mathrm{T}}$ and $D$. lykanthroporepellens $\mathrm{BL}-\mathrm{DC}-9^{\mathrm{T}}$, respectively. At higher initial 1,1,2-TCA concentrations, small amounts of vinyl chloride were also detected, but in amounts that were not statistically different from abiotic negative controls at a confidence level of $95 \%$.

Hydrogen $\left(\mathrm{H}_{2}\right)$ remained at relatively high concentrations $(>62 \%, v / v)$ in the gas headspace at the end of the eight-week incubation period for all chlorinated solvent concentrations tested for both strains, indicating that it was not stoichiometrically limiting.

\section{Discussion}

As a basis for comparing the concentrations tested here relative to saturation concentrations, solubility in water at $20^{\circ} \mathrm{C}$ is $86.1 \mathrm{mM}$ for 1,2-DCA (Horvath et al., 1999), $23.9 \mathrm{mM}$ for 1,2-DCP (Horvath et al., 1999), and 32.9 mM for 1,1,2-TCA (ATSDR 1989). Also as a basis for comparison, groundwater in the well from which $D$. lykanthroporepellens BL-DC- ${ }^{\mathrm{T}}$ was isolated had 1,2DCA, 1,2-DCP, and 1,1,2-TCA concentrations that averaged $5.5 \mathrm{mM}, 0.6 \mathrm{mM}$, and $2.8 \mathrm{mM}$, respectively (Bowman et al., 2006; Yan et al., 2009a). Results determined here indicate that both $D$. alkenigignens IP3- $3^{\mathrm{T}}$ and D. lykanthroporepellens BL-DC- $9^{\mathrm{T}}$ can reductively dehalogenate 1,2-DCA, 1,2-DCP, and 1,1,2-TCA at concentrations comparable to those present in the DNAPL source zone area of the Brooklawn area of the PPI site.

D. alkenigignens IP3-3 ${ }^{\mathrm{T}}$ was isolated from groundwater contaminated with 1,2-DCA, 1,2-DCP, and 1,1,2-TCA at concentrations of $0.023 \mathrm{mM}, 0.021 \mathrm{mM}$, and $0.010 \mathrm{mM}$, respectively (Bowman et al., 2012). Although initially isolated from an environment with much lower chlorinated solvent concentrations than D. lykanthroporepellens BL-DC- $-9^{\mathrm{T}}$, results from the present study demonstrate that $D$. alkenigignens IP3-3 ${ }^{\mathrm{T}}$ can reductively dechlorinate 1,2-DCA, 1,2-DCP, and 1,1,2-TCA at concentrations comparable to D. lykanthroporepellens BL-DC- $9^{\mathrm{T}}$.

Although reports of pure cultures' abilities to dehalogenate high concentrations of chlorinated alkanes are generally lacking in the literature, Marzorati et al. (2007) reported an enrichment culture referred to as 6VS (originating from groundwater in Italy where there was 1,2DCA contamination for more than 30 years) that repeatedly dechlorinated $8 \mathrm{mM}$ 1,2-DCA. Also, Grostern and Edwards (2009) described an enrichment culture, including Dehalobacter sp. and an Acetobacterium sp., capable of dechlorinating $2 \mathrm{mM}$ 1,2-DCA. Though not previously evaluated for chlorinated ethanes or propanes, previous research on chlorinated ethenes has shown that microbial populations reductively dechlorinating chlorinated aliphatic alkenes, particularly perchloroethene (PCE) and trichloroethene (TCE) can maintain their activity and increase contaminant dissolution rates (Cope and Hughes, 2001; Dennis et al., 2003; Sleep et al., 2006; Yang and McCarty 2002).

The toxicity of solvents to microorganisms has been previously correlated to hydrophobicity as measured by the $\log$ of octanol/water partition coefficients, log $\mathrm{K}_{\mathrm{ow}}$ (Sikkema et al., 1995). Compounds with $\log K_{o w}$ in the range of 1.5 to 4 are generally toxic to microorganisms, with maximum toxicity exhibited by compounds with $\log \mathrm{K}_{\mathrm{ow}}$ between 2 and 4 (Bowman et al., 2009; Inoue and Horikoshi, 1991; Kieboom and de Bont, 2000; Sikkema et al., 1994; Sikkema et al., 1995). The adverse effects of 1,2-DCA, 1,2-DCP, and 1,1,2-TCA on reductive dechlorination by the bacterial strains tested here are consistent with these previous observations. For equal molar concentrations, 1,1,2-TCA [ $\log \mathrm{K}_{\mathrm{ow}} 2.47$, (Alvarez and Illman, 2006)] was more inhibitory than 1,2-DCP [ $\log \mathrm{K}_{\mathrm{ow}} 2.0$, (Alvarez and Illman, 2006)] which had a larger adverse effect than 1,2-DCA $\left[\log \mathrm{K}_{\mathrm{ow}} 1.48\right.$, (Alvarez and Illman, 2006)].

The ability of Dehalogenimonas spp. to reductively dechlorinate high concentrations of halogenated alkanes has important implications for cleanup of contaminated soil and groundwater. Abiotic transformation of these chemicals in the environment is generally quite slow. For example, the environmental half-life of 1,2-DCA from abiotic transformation in water was estimated to be 50 years (Vogel et al., 1987). Unlike chlorinated ethenes (e.g., tetrachloroethene and trichloroethene), several of the polychlorinated ethanes and propanes, 1,2DCA in particular, are resistant to transformation by zero-valent iron (Sarathy et al., 2010; Song and Carraway, 2005), limiting physicochemical remediation approaches for cleanup. The fact that Dehalogenimonas spp. are able to perform reductive dechlorination even in the presence of high concentrations of chlorinated compounds suggests that they may provide an important role in bioremediation.

\section{Abbreviations}

1,2-DCA: 1,2-dichloroethane; 1,2-DCP: 1,2-dichloropropane; 1,1,2-TCA: 1,1,2-trichloroethane.

\section{Competing interests}

The authors declare that they have no competing interests. 


\section{Authors' contributions}

JY conducted preliminary experiments to assess the range of chlorinated solvents dehalogenated by D. lykanthroporepellens BL-DC-9 ${ }^{\top}$. ADM and KSB carried out the final experiments with $D$. alkenigignens IP3-3 ${ }^{\top}$ and $D$. lykanthroporepellens BL-DC-9 ${ }^{\top}$. WMM and FAR guided the research. All authors contributed to data interpretation and writing of the manuscript. All authors read and approved the final manuscript.

\section{Acknowledgements}

This research was funded by the Governor's Biotechnology Initiative of the Louisiana Board of Regents grant BOR\#015 and NPC Services, Inc.

\section{Author details}

${ }^{1}$ Department of Civil and Environmental Engineering, Louisiana State University, 3513B Patrick Taylor Hall, Baton Rouge, LA 70803, USA. 2Department of Biological Sciences, Louisiana State University, Baton Rouge, LA 70803, USA. ${ }^{3}$ Department of Biological Sciences, University of Alaska Anchorage, Anchorage, AK 99508, USA. ${ }^{4}$ Present address: Jun Yan, Department of Microbiology and Department of Civil and Environmental Engineering, University of Tennessee, Knoxville, TN, USA.

Received: 1 October 2012 Accepted: 2 October 2012 Published: 9 October 2012

\section{References}

Agency for Toxic Substances and Disease Registry (ATSDR) (2001) Toxicological profile for 1,2-dichloroethane. Public Health Service U.S. Department of Health and Human Services, Atlanta, GA, http://www.atsdr.cdc.gov/tfacts38. pdf. Accessed 11 August 2012

Agency for Toxic Substances and Disease Registry (ATSDR) (1989) Toxicological profile for 1,1,2-trichloroethane. Public Health Service U.S. Department of Health and Human Services, Atlanta, GA, http://www.atsdr.cdc.gov/ toxprofiles/tp148.pdf. Accessed 11 August 2012

Alvarez PJJ, IIIman WA (2006) Bioremediation and natural attenuation: Process fundamentals and mathematical models. Wiley, Hoboken, NJ

Bowman KS, Moe WM, Rash BA, Bae HS, Rainey FA (2006) Bacterial diversity of an acidic Louisiana groundwater contaminated by dense nonaqueous-phase liquid containing chloroethanes and other solvents. FEMS Microbiol Ecol 58:120-133. doi:10.1111/j.1574-6941.2006.00146.x

Bowman KS, Nobre MF, da Costa MS, Rainey FA, Moe WM (2012) Dehalogenimonas alkenigignens sp. nov, a chlorinated alkane dehalogenating bacterium isolated from groundwater. Int J Syst Evol Microbiol. doi:10.1099/ ijs.0.045054-0, in press

Bowman KS, Rainey FA, Moe WM (2009) Production of hydrogen by Clostridium species in the presence of chlorinated solvents. FEMS Microbiol Lett 290:188-194. doi:10.1111/j.1574-6968.2008.01419.x

Chen C, Puhakka JA, Ferguson JF (1996) Transformations of 1,1,2,2tetrachloroethane under methanogenic conditions. Environ Sci Technol 30:542-547. doi:10.1021/es9502987

Christ JA, Ramsburg CA, Abriola LM, Pennell KD, Löffler FE (2005) Coupling aggressive mass removal with microbial reductive dechlorination for remediation of DNAPL source zones: A review and assessment. Environ Health Perspect 113:465-477. doi:10.1289/ehp. 6932

Cope N, Hughes JB (2001) Biologically-enhanced removal of PCE from NAPL source zones. Environ Sci Technol 35:2014-2021. doi:10.1021/es0017357

De Wildeman S, Verstraete W (2003) The quest for microbial reductive dechlorination of $C_{2}$ to $C_{4}$ chloroalkanes is warranted. Appl Microbiol Biotechnol 61:94-102. doi:10.1007/s00253-002-1174-6

De Wildeman S, Diekert G, Van Langenhove H, Verstraete W (2003) Stereoselective microbial dehalorespiration with vicinal dichlorinated alkanes. Appl Environ Microbiol 69:5643-5647. doi:10.1128/AEM.69.9.5643-5647.2003

Dennis PC, Sleep BE, Fulthorpe RR, Liss SN (2003) Phylogenetic analysis of bacterial populations in an anaerobic microbial consortium capable of degrading saturation concentrations of tetrachloroethylene. Can J Microbiol 49:15-27. doi:10.1139/W03-008

Fennell DE, Carroll AB, Gossett JM, Zinder SH (2001) Assessment of indigenous reductive dechlorination potential at a TCE-contaminated site using microcosms, polymerase chain reaction analyses and site data. Environ Sci Technol 35:1830-1839. doi:10.1021/es0016203
Field JA, Sierra-Alvarez R (2004) Biodegradability of chlorinated solvents and related chlorinated aliphatic compounds. Reviews in Environ Sci \& Bio/ Technol 3:185-254. doi:10.1007/s11157-004-4733-8

Fletcher KE, Löffler FE, Richnow HH, Nijenhuis I (2009) Stable carbon isotope fractionation of 1,2-dichloropropane during dichloroelimination by Dehalococcoides populations. Environ Sci Technol 43:6915-6919. doi:0.1021/ es $900365 x$

Grostern A, Edwards EA (2006) Growth of Dehalobacter and Dehalococcoides spp. during degradation of chlorinated ethanes. Appl Environ Microbiol 72:428436. doi:10.1128/AEM.72.1.428-436.2006

Grostern A, Edwards EA (2009) Characterization of a Dehalobacter coculture that dechlorinates 1,2-dichloroethane to ethene and identification of the putative reductive dehalogenase gene. Appl Environ Microbiol 75:2684-2693. doi:10.1128/AEM.02037-08

He J, Sung Y, Krajmalnik-Brown R, Ritalahti KM, Löffler FE (2005) Isolation and characterization of Dehalococcoides sp. strain FL2, a trichloroethene (TCE) and 1,2-dichloroethene-respring anaerobe. Environ Microbiol 7:1442-1450. doi:10.1111/j.1462-2920.2005.00830.x

Horvath AL, Gerzen FW, Maczynska Z (1999) IUPAC-NIST solubility data series. J Phys Chem Ref Data 28:395-626. doi:10.1063/1.556039

Inoue A, Horikoshi K (1991) Estimation of solvent-tolerance of bacteria by the solvent parameter log P. J Ferment Bioeng 71:194-196. doi:10.1016/0922338X(91)90109-T

Kieboom J, de Bont JAM (2000) Mechanisms of organic solvent resistance in bacteria. In: Storz G, Hengge-Aronis R (eds) Bacterial stress responses. ASM Press, Washington, DC, pp 393-402

Lorah M, Olsen L (1999) Natural attenuation of chlorinated volatile organic compounds in a freshwater tidal wetland: Field evidence of anaerobic biodegradation. Water Resources Res 35:3811-3827. doi:10.1029/ 1999WR900116

Löffler FE, Champine JE, Ritalahti KM, Sprague SJ, Tiedje JM (1997) Complete reductive dechlorination of 1,2-dichloropropane by anaerobic bacteria. Appl Environ Microbiol 63:2870-2875

Löffler FE, Yan J, Ritalahti KM, Adrian L, Edwards EA, Konstantinidis KT, Müller JA, Fullerton H, Zinder SH, Spormann AM (2012) Dehalococcoides mccartyi gen. nov., sp. nov., obligate organohalide-respiring anaerobic bacteria, relevant to halogen cycling and bioremediation, belong to a novel class, Dehalococcoidestes classis nov., within the phylum Chloroflexi. Int J Syst Evol Microbiol. doi:10.1099/ijs.0.034926-0, in press

Major DW, McMaster ML, Cox EE, Edwards EA, Dworatzek SM, Hendrickson ER, Starr MG, Payne JA, Buonamici LW (2002) Field demonstration of successful bioaugmentation to achieve dechlorination of tetrachloroethene to ethene. Environ Sci Technol 36:5106-5116. doi:10.1021/es0255711

Marzorati M, de Ferra F, Van Raemdonck H, Borin S, Allifranchini E, Carpani G, Serbolisca L, Verstraete W, Boon N, Daffonchio D (2007) A novel reductive dehalogenase, identified in a contaminated groundwater enrichment culture and in Desulfitobacterium dichloroeliminans strain DCA1 is linked to dehalogenation of 1,2-dichloroethane. Appl Environ Microbiol 73:2990-2999. doi:10.1128/AEM.02748-06

Maymó-Gatell X, Anguish T, Zinder SH (1999) Reductive dechlorination of chlorinated ethenes and 1,2-dichloroethane by "Dehalococcoides ethenogenes" 195. Appl Environ Microbiol 65:3108-3113

Moe WM, Yan J, Nobre MF, da Costa MS, Rainey FA (2009) Dehalogenimonas lykanthroporepellens gen. nov., sp. nov., a reductive dehalogenating bacterium isolated from chlorinated solvent contaminated groundwater. Int J Syst Evol Microbiol 59:2692-2697. doi:10.1099/ijs.0.011502-0

Sarathy V, Salter AJ, Nurmi J, Johnson GO, Johnson RL, Tratnyek PG (2010) Degradation of 1,2,3-trichloropropane (TCP): Hydrolysis, elimination, and reduction by iron and zinc. Environ Sci Technol 44:787-793. doi:10.1021/es902595j

Sikkema J, de Bont JAM, Poolman B (1994) Interactions of cyclic hydrocarbons with biological membranes. J Biol Chem 174:8022-8028

Sikkema J, de Bont JAM, Poolman B (1995) Mechanism of membrane toxicity of hydrocarbons. Microbiol Rev 59:201-222

Sleep BE, Seepersad DJ, Mo K, Heidorn CM, Hrapovic L, Morrill PL, McMaster ML, Hood ED, Lebron C, Lollar BS, Major DW, Edwards EA (2006) Biological enhancement of tetrachloroethene dissolution and associated microbial community changes. Environ Sci Technol 40:3623-3633. doi:10.1021/ es051493g

Song H, Carraway ER (2005) Reduction of chlorinated ethanes by nanosized zerovalent iron: Kinetics, pathways, and effects of reaction conditions. Environ Sci Technol 39:6237-6245. doi:10.1021/es048262e 
van Ginkel S, Sung S, Lay JJ (2001) Biohydrogen production as a function of pH and substrate concentration. Environ Sci Technol 35:4726-4730. doi:10.1021/ es001979r

Vogel TM, Criddle CS, McCarty PL (1987) Transformations of halogenated aliphatic compounds. Environ Sci Technol 21:722-736. doi:10.1021/ es00162a001

Yan J, Rash BA, Rainey FA, Moe WM (2009a) Isolation of novel bacteria within the Chloroflexi capable of reductive dechlorination of 1,2,3-trichloropropane. Environ Microbiol 11:833-843. doi:10.1111/j.1462-2920.2008.01804.x

Yan J, Rash BA, Rainey FA, Moe WM (2009b) Detection and quantification of Dehalogenimonas and "Dehalococcoides" populations via PCR-based protocols targeting 16S rRNA genes. Appl Environ Microbiol 75:7560-7564. doi:10.1128/ AEM.01938-09

Yang YR, McCarty PL (2002) Comparison between donor substrates for biologically enhanced tetrachloroethene DNAPL dissolution. Environ Sci Technol 36:3400-3404. doi:10.1021/es011408e

doi:10.1186/2191-0855-2-54

Cite this article as: Maness et al:: Dehalogenimonas spp. can

Reductively Dehalogenate High Concentrations of 1,2-Dichloroethane,

1,2-Dichloropropane, and 1,1,2-Trichloroethane. AMB Express 2012 2:54.

\section{Submit your manuscript to a SpringerOpen ${ }^{\circ}$} journal and benefit from:

- Convenient online submission

- Rigorous peer review

- Immediate publication on acceptance

- Open access: articles freely available online

- High visibility within the field

- Retaining the copyright to your article

Submit your next manuscript at $\gg$ springeropen.com 